\title{
Analyses of the mitochondrial mutations in the Chinese patients with sporadic Creutzfeldt-Jakob disease
}

\author{
Jin Zhang ${ }^{1,2}$, Zhi-Xia Zhang ${ }^{1}$, Peng-Chen $\mathrm{Du}^{3}$, Wei Zhou ${ }^{1}$, Su-Dong $\mathrm{Wu}^{4}$, Qi-Ling Wang ${ }^{5}$, Cao Chen ${ }^{1}$, \\ Qi Shi ${ }^{1}$, Chen $\mathrm{Chen}^{3}$, Chen Gao ${ }^{1}$, Chan Tian ${ }^{1}$ and Xiao-Ping Dong ${ }^{\star, 1,6}$
}

Pathogenic mitochondrial DNA (mtDNA) mutations leading to mitochondrial dysfunction can cause a variety of chronic diseases in central nervous system (CNS). However, the role of mtDNA mutations in sporadic Creutzfeldt-Jakob disease (sCJD) has still been unknown. In this study, we comparatively analyzed complete mtDNA sequences of 31 Chinese sCJD patients and 32 controls. Using MITOMASTER and PhyloTree, we characterized 520 variants in SCJD patients and 507 variants in control by haplogroup and allele frequencies. We classified the mtDNAs into 40 sub-haplogroups of 5 haplogroups, most of them being Asian-specific haplogroups. Haplogroup U, an European-specific haplogroups mtDNA, was found only in SCJD. The analysis to control region (CR) revealed a $31 \%$ increase in the frequency of mtDNA CR mutations in SCJD versus controls. In functional elements of the mtDNA CR, six CR mutations were in conserved sequence blocks I (CSBI) in SCJD, while only one in control $(P<0.05)$. More mutants in transfer ribonucleic acid-Leu (tRNA-Leu) were detected in SCJD. The frequencies of two synonymous amino-acid changes, m.11467A $>$ G, p. $(=)$ in NADH dehydrogenase subunit 4 (ND4) and m.12372G $>$ A, p. $(=)$ in NADH dehydrogenase subunit 5 (ND5), in SCJD patients were higher than that of controls. Our study, for the first time, screened the variations of mtDNA of Chinese SCJD patients and identified some potential disease-related mutations for further investigations.

European Journal of Human Genetics (2015) 23, 86-91; doi:10.1038/ejhg.2014.52; published online 26 March 2014

\section{INTRODUCTION}

Creutzfeldt-Jakob disease (CJD), the most common form of transmissible spongiform encephalopathies (TSEs) or prion disease in human beings, is characterized by the aggregation of partially protease-resistant isoform $\mathrm{PrP}^{\mathrm{Sc}}$ and spongiform degeneration in the central nervous system (CNS). The most common form of CJD ( $>85 \%$ ) is sporadic CJD (sCJD), which occurs with an incidence of about 1-2 per million individuals per year. ${ }^{1}$ It is considered to be spontaneous because no epidemiological evidence for association with any exogenous factors.

Although up to now the aetiology of sCJD remains unknown, many researchers have proposed that it has powerful genetic determinants. $^{2}$ Studies demonstrate a common polymorphism in the coding region of the PrP gene locus gene (PRNP) at codon 129 is a well-established genetic risk factor for $\mathrm{sCJD}^{3}$ Homozygosity at PRNP codon 129 increases the risk of sCJD in Caucasian. ${ }^{4}$ Codons 127 and 219 of $P R N P$ also harbour amino-acid (AA) polymorphisms that confer resistance to $\mathrm{Kuru}^{5}$ or sCJD. ${ }^{6}$ Recently, other candidate genes have been identified as risk factors for sCJD, such as an SNP upstream of PRNP exon 1 (SNP 1368), ${ }^{7}$ c.592C $>\mathrm{T}(\mathrm{p} . \mathrm{T} 174 \mathrm{M})$ in prion-like doppel gene $(P R N D),{ }^{8} A P O E \varepsilon 4$ allele, ${ }^{9}$ polymorphisms at CALHM1 gene, ${ }^{10}$ and BACE1 gene. ${ }^{11}$ Identification of the potential genetic risk factors for sCJD seems to be one of the important pathways for understanding of the pathogenic mechanisms and human susceptibility to the disease.

The mitochondrion is the essential organelle that provides energy in the form of ATP for normal cell function. ${ }^{12}$ Although $>95 \%$ of all enzymes present in the mitochondria are encoded by the nuclear genome, mitochondrial DNA (mtDNA), a 16 569-bp circle of doublestranded DNA in mitochondria contains 37 genes specifying: 13 polypeptides, 22 transfer ribonucleic acids (tRNAs), and 2 ribosomal ribonucleic acids (rRNAs). ${ }^{13}$ The rate of sequence evolution in mtDNA is 10-20 times higher than that in the nuclear genome. ${ }^{14}$ Thereby mutations in mtDNA are the major reason for the abnormalities of mitochondrion.

Mutations in the mtDNA can result in defect of mitochondrial oxidative phosphorylation (OXPHOS), which may subsequently inhibit ATP production, exacerbate generation of reactive oxygen species (ROS), affect calcium homeostasis, and induce apoptosis. ${ }^{15}$ High levels of ROS cause damage of cell membranes through lipid peroxidation and accelerate the high mutation rate of mtDNA. Accumulation of mtDNA mutations enhances oxidative damage, causes energy depletion, and increases ROS production, in a vicious cycle. ${ }^{16}$ Moreover, the brain is especially prone to oxidative

${ }^{1}$ State Key Laboratory for Infectious Disease Prevention and Control, Collaborative Innovation Center for Diagnosis and Treatment of Infectious Diseases (Zhejiang University), National Institute for Viral Disease Control and Prevention, Chinese Center for Disease Control and Prevention, Beijing, People's Republic of China; ${ }^{2}$ Shandong International Travel Health Care Center, Shandong Entry-Exit Inspection and Quarantine Bureau, Qingdao, People's Republic of China; ${ }^{3}$ State Key Laboratory for Infectious Disease Prevention and Control, National Institute for Communicable Disease Control and Prevention, Chinese Center for Disease Control and Prevention, Beijing, People's Republic of China; ${ }^{4}$ Department of Oncology, Chinese Navy General Hospital, Beijing, People's Republic of China; ${ }^{5}$ Key Laboratory of Male Reproduction and Genetics, National Health and Family Planning Commission of China, Guangzhou, People's Republic of China; ${ }^{6}$ Chinese Academy of Sciences Key Laboratory of Pathogenic Microbiology and Immunology, Institute of Microbiology, Chinese Academy of Sciences, Beijing, People's Republic of China

${ }^{*}$ Correspondence: Dr X-P Dong, National Institute for Viral Disease Control and Prevention, Chinese Center for Disease Control and Prevention, Changbai Road 155, Beijing 102206, People's Republic of China. Tel: +86 10 58900815; Fax: +86 10 58900815; E-mail: dongxp238@sina.com

Received 22 August 2013; revised 18 February 2014; accepted 19 February 2014; published online 26 March 2014 
stress-induced damage due to its high levels of polyunsaturated fatty acids, high oxygen consumption, high content in transition metals, and poor antioxidant defenses. ${ }^{17}$ There is increasing evidence that impairment of mitochondrial energy metabolism has an important role in the pathophysiology of some chronic brain disorders and the mutations in mtDNA are now recognized as the major contributors to brain disorders, such as Leber's hereditary optic neuropathy (LHON), ${ }^{18}$ mitochondrial encephalomyopathy, ${ }^{19}$ and Alzheimer's disease (AD). ${ }^{20}$ Transition at $\mathrm{np} 4336$ of the mitochondrial tRNA glutamine gene is a risk factor for $\mathrm{AD} .{ }^{21}$ Since CJD shares similar pathological changes with $\mathrm{AD}$, including abnormal protein deposits in CNS tissues, faulty calcium metabolism, high level of ROS and apoptosis in neurons, it is reasonable to assume a role of mtDNA variants in SCJD.

To date, the situation of mtDNA mutations is rarely described in the patients with sCJD. To explore the potential association of mtDNA mutations or well-known polymorphisms in the control and coding regions with $\mathrm{sCJD}$, a mutational screening of the mtDNA sequences of 31 sCJD patients and 32 non-CJD cases at the ages from 45 to 55 years old was carried out. Analyses of mtDNA sequences in those subjects identified a total of $1131 \mathrm{mtDNA}$ different variants in sCJD patients compared with the revised Cambridge Reference Sequence (rCRS). These variants have been further evaluated by phylogenetic analysis, structure function relation and our efforts were made to establish the relationship of each variant with sCJD.

\section{MATERIALS AND METHODS}

Ethics statement

Usage of the stored human peripheral blood samples in China CJD Surveillance System has been approved by the Ethical Committee of National Institute for Viral Disease Prevention and Control, China CDC.

\section{Clinical samples}

The peripheral blood samples from 31 Chinese probable sCJD cases and 32 non-CJD cases that did not fulfill the criteria for CJD were included in this study. The diagnoses of CJD were made by China CJD Surveillance Centre according to WHO CJD diagnostic criteria. The diagnosis for each case was made by an expert board consisting of neurologists, neuropathologists, epidemiologists, and laboratory staff. Peripheral blood samples were collected in EDTA-containing vacutainer tubes and stored at $-80^{\circ} \mathrm{C}$ until DNA extraction. DNA was extracted by using the Qiagen's DNA purification kit (Hilden, Germany) according to the manufacturer's instructions

\section{PCR Amplification of mtDNA}

The whole mitochondrial genome of each tested sample was individually amplified with 24 pairs of primers. PCR amplifications for all primer sets were performed in a $25-\mu \mathrm{l}$ volume. The PCR products were kept at $-20^{\circ} \mathrm{C}$ until sequencing. The full mtDNA genome was sequenced. All fragments were sequenced forward and backward for confirmation of any nucleotide variation.

\section{Sequence analysis using MITOMASTER}

We assembled the data for both sCJD and non-CJD groups using Jellyfish $\mathrm{v}$ 1.3.1 (Field Scientific LLC, Lewisburg, PA, USA), a sequence analysis software in comparison with mtDNA reference, the rCRS (NC_012920.1) provided by the National Center. The complete mtDNA sequence of each case was analyzed by MITOMASTER (http://www.mitomap.org), which included a database of over $3600 \mathrm{mtDNA}$ sequences from NCBI GenBank. Using MITOMASTER, the mtDNA sequence of each case was compared with the rCRS and all mtDNA variants identified. The array of sequence variants was used to determine the haplogroup of the tested case. The haplogroup-associated variants were identified and the population frequency of each nucleotide sequence variant was calculated relative to the MITOMASTER and Pereira's databases. To identify rare variants, we arbitrarily defined variants with allele frequencies
$<0.5 \%$ as rare and those with frequencies $>0.5 \%$ as common, similar to cutoff used for nDNA variation. We have submitted our data to Mitomap database (http://www.mitomap.org/bin/view.pl/MITOMAP/VariantSubmissionList).

\section{Prediction of pathogenicity}

For prediction of pathogenic characteristics of all non-synonymous mtDNA changes, two analysis tools were used, Polymorphism Phenotyping v2 (PolyPhen-2) and Sorting Intolerant From Tolerant (SIFT). Polyphen scores of $>0.50$ are intolerant (possibly damaging). The scores of $<0.50$ are likely tolerant (benign). SIFT is based on the premise that protein evolution is correlated with protein function. ${ }^{22,23}$ SIFT scores range from 0 to 1 . The AA substitution is predicted to be damaging if the score is $\leq 0.05$, and to be tolerated if the score is $>0.05$.

\section{Statistical analysis}

A case-control association study was carried out between SCJD and non-CJD groups individually for each mtDNA mutation and for Haplogroup. Frequencies between SCJD cases and controls were compared to assess the associations of individual mtDNA mutations and Haplogroup using the Chi-square test. The $P$-value of $<0.05$ was considered as significant difference. Effect size for the association was measured as an odds ratio (OR) with a 95\% confidence interval (CI). A permutation test was employed to address the issue of multiple testing by Bonferroni. The statistical analyses were carried out using the statistical packages Stata SE12.0 (StataCorp LP, College Station, TX, USA).

\section{RESULTS}

mtDNA sequence variants detected by sequencing relative to rCRS mtDNA sequencing following the amplification of whole mtDNA revealed a total of $1131 \mathrm{mtDNA}$ variants in $31 \mathrm{sCJD}$ patients and 1189 mtDNA variants in 32 non-CJD cases compared with the rCRS (Supplementary Table 1). On the basis of both coding- and controlregion mutations, including haplogroup nomenclature, mtDNAs from patients and controls were classified into 40 sub-haplogroups of 5 haplogroups followed a phylogenetic tree of global human mtDNA variation. Most of them were typical of modern East Eurasian populations, while a few were West Eurasian (R0 and U) mtDNAs. The only haplogroup that showed statistical association with sCJD patients was haplogroup $\mathrm{U}$, that four sCJD patients, but none of nonCJD cases, belonged to haplogroup $\mathrm{U}$, with a $P$-value of 0.035 (Table 1). However, this significance was not maintained after correcting for multiple hypotheses using a permutation test procedure (adjusted $P$-value $=0.056$ )

Table 1 Frequencies of mtDNA haplogroups in the groups of sCJD and controls

\begin{tabular}{|c|c|c|c|c|c|c|c|c|}
\hline \multirow[b]{2}{*}{ Haplogroup ${ }^{a}$} & \multicolumn{2}{|c|}{$\begin{array}{c}\text { SCJD } \\
(\mathrm{n}=31)\end{array}$} & \multicolumn{2}{|c|}{$\begin{array}{c}\text { Ctrl } \\
(\mathrm{n}=32)\end{array}$} & \multirow{2}{*}{\multicolumn{2}{|c|}{$\mathrm{CHI} 2$}} & \multirow[b]{2}{*}{ Adjusted } & \multirow[b]{2}{*}{ OR $(95 \% \mathrm{Cl})$} \\
\hline & $\mathrm{n}$ & $\%$ & $n$ & $\%$ & & & & \\
\hline$U^{b}$ & 4 & 12.9 & 0 & 0 & 4.409 & 0.035 & 0.056 & - \\
\hline RO & 1 & 3.22 & 1 & 3.13 & 0.0005 & 0.98 & 1 & $0.97(0.06-16.2)$ \\
\hline $\mathrm{R}$ & 4 & 12.9 & 10 & 31.25 & 1.9846 & 0.079 & 0.236 & $2.36(0.7-7.96)$ \\
\hline N & 7 & 22.58 & 2 & 6.25 & 3.4294 & 0.064 & 0.082 & $0.23(0.04-1.2)$ \\
\hline D & 5 & 16.13 & 8 & 25 & 1.4943 & 0.38 & 0.339 & $2.25(0.6-8.42)$ \\
\hline M & 10 & 32.26 & 11 & 34.38 & 0.0318 & 0.86 & 1 & $1.1(0.38-3.14)$ \\
\hline
\end{tabular}

a Haplogroups were grouped according to PhyloTree. $\mathrm{U}$ includes U2e and U4b; RO includes RO and $\mathrm{H} 1 \mathrm{bH}$; R includes B4d, B5a, F1, F1a, F1b, F3a, and J1c; N includes N9a, A4e, and W3a; D includes D4, D4a, D4b, D4c, D4e, D4i, D4j, D4k, and D5c; M includes M, M1, M7b, M7c, M8a, M9a, C4a, C7, C7a, and Za.

bStatistical difference between the groups of SCJD and control with a $P$-value of 0.035 , Statistical difference betwe $P$-value of 0.056

${ }^{c}$ Adjusted $P$-value: adjustment of Chi-square $P$-values was carried out with a permutation-based approach; number of permutations $=20000$. 
Excluding highly polymorphic mtDNA variants in the mtDNA control region (CR) and variants belonged to the mitochondrial haplogroup-specific variants (Supplementary Table 2), the rest of 520 mtDNA variants in $31 \mathrm{sCJD}$ patients, 285 different kinds of mtDNA variants were identified (Supplementary Figure 1).

\section{Novel non-haplogroup-associated mtDNA variants}

Haplogroup-associated variation accounted for most of the 520 substitution variants (Supplementary Table 3). On the basis of allele frequencies, there were $36(13 \%)$ novel mtDNA variants. Among them, 13 were mapped in the protein-coding regions. Excluding two variants $(\mathrm{m} .6228 \mathrm{C}>\mathrm{T}$ and $\mathrm{m} .14067 \mathrm{C}>\mathrm{T})$ that were haplogroupassociated ones, four variants were non-haplogroup-associated variants leading to synonymous AA changes and seven were nonsynonymous variants (Table 2). Four altered AAs out of those seven variants seemed to be able to change the conservative AAs $(\mathrm{CI}>87 \%$; Table 2). SIFT and PolyPhen analyses proposed that three of them were possibly pathogenic changes. Two variants, m.11375A $>C$ (p.K206Q in NADH dehydrogenase subunit 4 (ND4)) and m.12631T $>$ A (p.S99T in NADH dehydrogenase subunit 5 (ND5)), were observed in the case 9 .

Identification and quantification of the mtDNA variants in the $\mathrm{CR}$ To determine the presence of mtDNA mutations in the CR (nps 1_578), the mutants in this region which involved in the known functional elements of the mtDNA CR were selected (Figure 1a). Generally, the frequency of mtDNA CR mutations in the group of sCJD was about $31 \%$ increased compared with that of non-CJD (Figure 1b). Regarding the functional elements of the mtDNA CR, there were six CR mutations in conserved sequence blocks I (CSBI) in the sCJD patients, but only one in the non-CJD cases $(P<0.05)$, while no obvious difference in the other functional elements was noticed between two groups (Figure 1c). It seems that the group of sCJD patients has more mutations in the functional elements of mtDNA $\mathrm{CR}$, which may influence the biological functions of those elements.

\section{Identification and quantification of the mtDNA variants in the regions for tRNA and rRNA}

The mtDNA sequencings identified 40 and 20 nucleotide variations within tRNA genes in the groups of sCJD and non-CJD, respectively (Figure 2). More mutants in tRNA-Leu were observed in the sCJD patients. Especially an A to $G$ transition mutation at position of 12308 , which located in the variable loop of tRNA-Leu, was found in five tested SCJD cases, but not in all non-CJD cases, showing statistical difference in the frequency of m.12308A $>\mathrm{G} \quad(P-$ value $=0.018$, adjusted $P$-value $=0.024)$. The number of mutations within rRNA gene between sCJD and controls were comparable without statistical difference (Supplementary Figure 2).

\section{Case-control association for variations in protein-coding genes}

There were 108 variants leading to non-synonymous AA changes in protein coding in those two groups. Frequency of non-synonymous sequence variations in all genes did not reveal statistically significant between sCJD patients and controls (Supplementary Table 4). All nucleotide variations identified in the current study were homoplasmic (Supplementary Table 1).

SIFT and PolyPhen analyses of all non-synonymous changes from sCJD and non-CJD revealed 18 pathogenic changes, without statistical difference between sCJD and non-CJD groups (Supplementary Table 5). Remarkably, two synonymous AA changes c.11467A $>$ G, p. $(=)$ in ND4 and c.12372G $>$ A, p. $(=)$ in ND5 showed higher frequencies in the sCJD patients with statistically different from the non-CJD cases. These two mutants have been reported to be related with altered brain $\mathrm{pH}$ value.

\section{DISCUSSION}

In prion diseases, the impairment of mitochondrial function is repeatedly observed, which could potentially contribute to or even initiate the various abnormalities, for example, synaptic pathology, ${ }^{24}$ faulty calcium metabolism, ${ }^{25}$ high level of ROS, ${ }^{26}$ and apoptosis of neurons. ${ }^{27}$ However, it has still been unclear whether mtDNA mutations as a risk factor for sCJD. In this study, we have screened and compared $31 \mathrm{sCJD}$ cases and 32 controls for mtDNA variations. We have found that haplogroup $U$ appears to be closely associated with Chinese SCJD, though this significance was not maintained after correcting for multiple hypotheses. This finding is somehow unexpected, since European-specific haplogroup U is uncommon in populations of China. The haplogroup-related SNPs may relate to partial uncoupling of OXPHOS and decreased efficiency of ATP production. ${ }^{28,29}$ This means that each haplogroup, with its different set of SNPs, can have unique bioenergetic properties and responses to oxidative stressors. The risks of developing $\mathrm{PD}$ and $\mathrm{AD}$ within the Western European are higher among mtDNA haplogroup $\mathrm{H}$, but lower for haplogroups $\mathrm{J}$ and $\mathrm{K} .{ }^{30,31}$ It is possible that the haplogroup $\mathrm{H}$ has significantly higher mitochondrial oxidative damage and higher VO (2max) (oxygen consumption) to produce more ROS. ${ }^{32}$ It seems to be that the association between haplogroup $\mathrm{U}$ and the risks of sCJD needs further assays in a larger sample scale.

Table 2 Eleven novel non-haplogroup-associated mitochondrial DNA variants in the protein-coding regions

\begin{tabular}{|c|c|c|c|c|c|c|c|}
\hline Location/Effect & Variant & Gene & AA change & $\mathrm{Cl}$ & Haplogroup & Case no. & Pathogenic \\
\hline \multirow[t]{4}{*}{ mRNA/Synon } & $\mathrm{m} .9732 \mathrm{C}>\mathrm{T}$ & $\operatorname{cox} 1$ & Silent & 0.98 & $M 7 b$ & 2 & \\
\hline & $\mathrm{m} .9974 \mathrm{C}>\mathrm{T}$ & COX3 & Silent & 0.98 & G1c & 15 & \\
\hline & m.11407C > T & ND4 & Silent & 1 & M7b & 2 & \\
\hline & $\mathrm{m} .13821 \mathrm{C}>\mathrm{T}$ & ND5 & Silent & 0.07 & N9a & 10 & \\
\hline \multirow[t]{7}{*}{ mRNA/Nonsyn } & m.11375A $>C$ & ND4 & p.K206Q & 1 & N9a & 9 & Possibly damaging \\
\hline & m. $12473 C>T$ & ND5 & p.146S & 0.47 & N9a, W3a & 9,11 & Possibly damaging \\
\hline & $\mathrm{m} .12631 \mathrm{~T}>\mathrm{A}$ & ND5 & p.S99T & 0.93 & N9a, W3a & 9,11 & Possibly damaging \\
\hline & m.14457T >C & ND6 & p.M73V & 0.89 & U4b & 29 & Benign \\
\hline & m.14753C $>T$ & СYTB & p.P3S & 0.2 & U2e, U2e & 21,24 & Benign \\
\hline & m.14980C $>A$ & CYTB & p.178M & 0.4 & G1c & 15 & Benign \\
\hline & m.15765G $>A$ & СYTB & p.G340E & 0.87 & B4d & 4 & Benign \\
\hline
\end{tabular}

Abbreviations: AA, amino acid; Cl, conservation index; COX1, cytochrome c oxidase I; COX3, cytochrome c oxidase III; CYTB, cytochrome B; Nonsyn, non-synonymous; Synon, synonymous. 
a

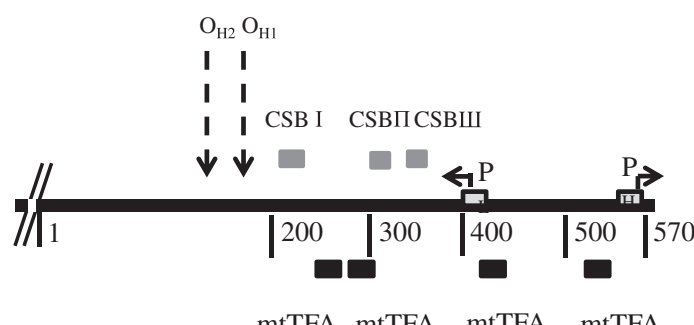

b

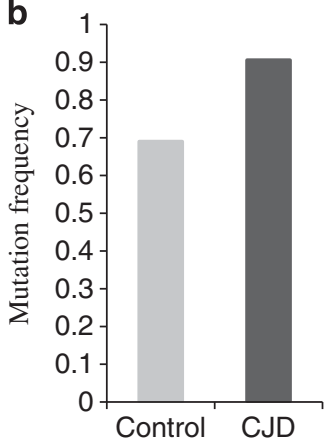

C

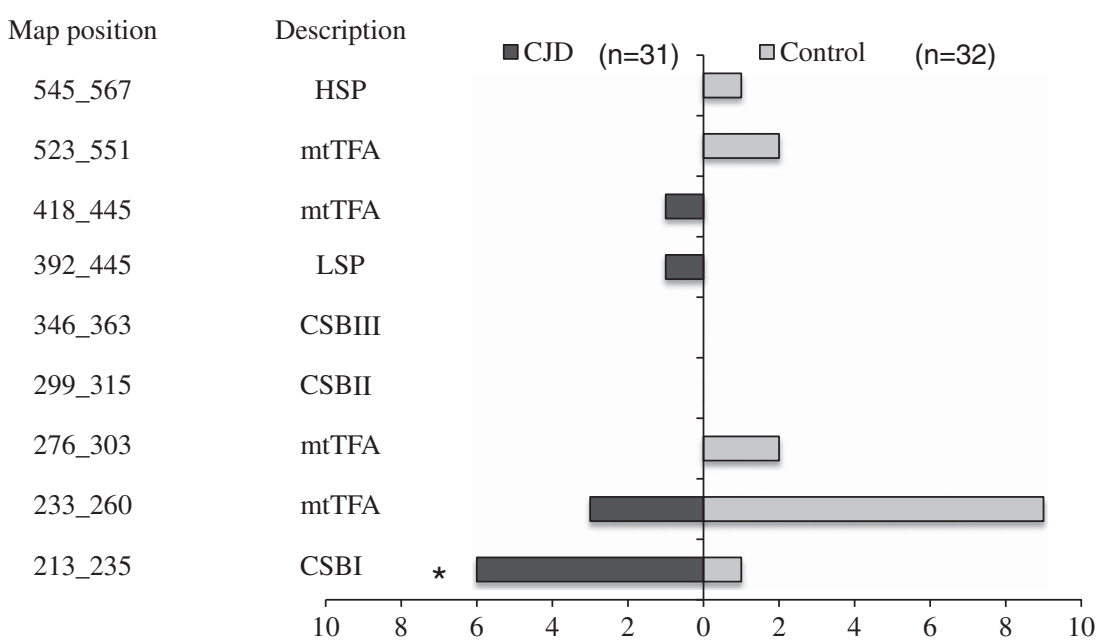

Figure 1 The numbers of the mutations in mtDNA control region (CR) regulatory elements in the groups of sCJD and control. (a) Schematic structure of a 570-bp CR in mtDNA. The CR includes the L- and $\mathrm{H}$-strand promoters (PL and PH), the binding sites of mitochondrial transcription factor A (mtTFA), the downstream conserved sequence blocks (CSB) I, II, and III, and the origins of $\mathrm{H}$-strand replication $(\mathrm{OH} 1$ and $\mathrm{OH} 2$ ). (b) mtDNA CR mutation frequency in the groups of SCJD and control. (c) The numbers of heteroplasmic mutations in mtDNA CR regulatory elements in the groups of sCJD and control. * $<0.05$.

On the basis of the review of the relevant literatures and websites, we have found 36 novel mtDNA variants in sCJD patients. Two of them, m.11375A > C (p.K206Q in ND4) and m.12631T >A (p.S99T in ND5), seem to be pathogenic mutations after assays by a couple of bioinformatics methods. ND4 and ND5 are two of seven subunits of complex I. Studies point out mutations in complex I genes defect in respiration, ATP synthesis, increase ROS production, and are associated with $\mathrm{LHON}^{33}$ Leigh's syndrome, ${ }^{34}$ mitochondrial encephalomyopathy, ${ }^{35}$ lactic acidosis stroke-like episodes (MELAS), and infertility. The exact effect on mitochondrial function of these two mutations deserves further study.

By analyzing the mtDNA CR sequence variation of sCJD and non-CJD, we conclude that SCJD patients display a high frequency of mtDNA CR mutations in the key elements of CSBI. Mutations in CSBI can result in the reduction of mtDNA copy numbers, because the L-strand transcript, processed at CSBI, has been proposed to provide the primer for initiating mtDNA $\mathrm{H}$-strand synthesis at $\mathrm{H}$-strand replication $1(\mathrm{OH} 1)$ and $\mathrm{H}$-strand replication $2(\mathrm{OH} 2)^{36}$ Lower mtDNA copy numbers will reduce the amounts of the encoding mitochondrial proteins, and subsequently affect the activities of complexes I, III, IV, and V.

The tRNA-Leu in SCJD patients have more mutations than in nonCJD cases, especially, the mutation of m.12308A $>G$ in the variable loop of tRNA-Leu. The tRNA-Leu encodes the most represented AA in the mitochondrial respiratory chain, suggesting a key role of this tRNA in mtDNA-coded OXPHOS subunits. The m.12308A $>\mathrm{G}$ variation has been reported associated with increased ROS production. The m.12308A $>\mathrm{G}$ change is a well-known polymorphism defined for European haplogroup $U$ and may constitute a risk factor for occipital stroke in patients with migraine, ${ }^{37} \mathrm{AD}$ in $\mathrm{man},{ }^{31}$ and severe knee osteoarthritis $(\mathrm{OA}){ }^{38}$ The association between m.12308A $>\mathrm{G}$ variation and the risks of sCJD needs further assays in a larger sample scale.

Two synonymous AA changes, m.11467A $>\mathrm{G}$ in ND4 and m.12372G $>A$ in ND5, are more frequently observed in sCJD patients. These two mutants are believed to associate with the $\mathrm{pH}$ alteration in brains, which may induce a significantly higher $\mathrm{pH}$ value $(7.006 \pm 0.18 \mathrm{SD})$ in brain tissues compared with that of control $(6.86 \pm 0.18 \mathrm{SD}) .{ }^{39}$ It has been hypothesized that these two mutants lead to loosing coupling due to less excess mitochondrial oxidation and decreased $\mathrm{H}+$ ion gradients in the outer membrane. The roles of those two mutations in the pathogenesis of prion diseases remain unsettled.

In summary, our study, for the first time, has screened mtDNA sequence variations in sCJD patients. sCJD patients have more mutations in CSBI versus controls. Mutants in tRNA-Leu, especially the frequency of m.12308A $>\mathrm{G}$ in sCJD patients, have statistical significance. European-specific haplogroup $U$ appears to be closely associated with Chinese sCJD. Two synonymous AA changes, m.11467A $>\mathrm{G}$ in ND4 and m.12372G $>A$ in ND5, show higher frequency in sCJD patients. The exact association between these mtDNA variations and the risks of sCJD needs further detailed assays. 


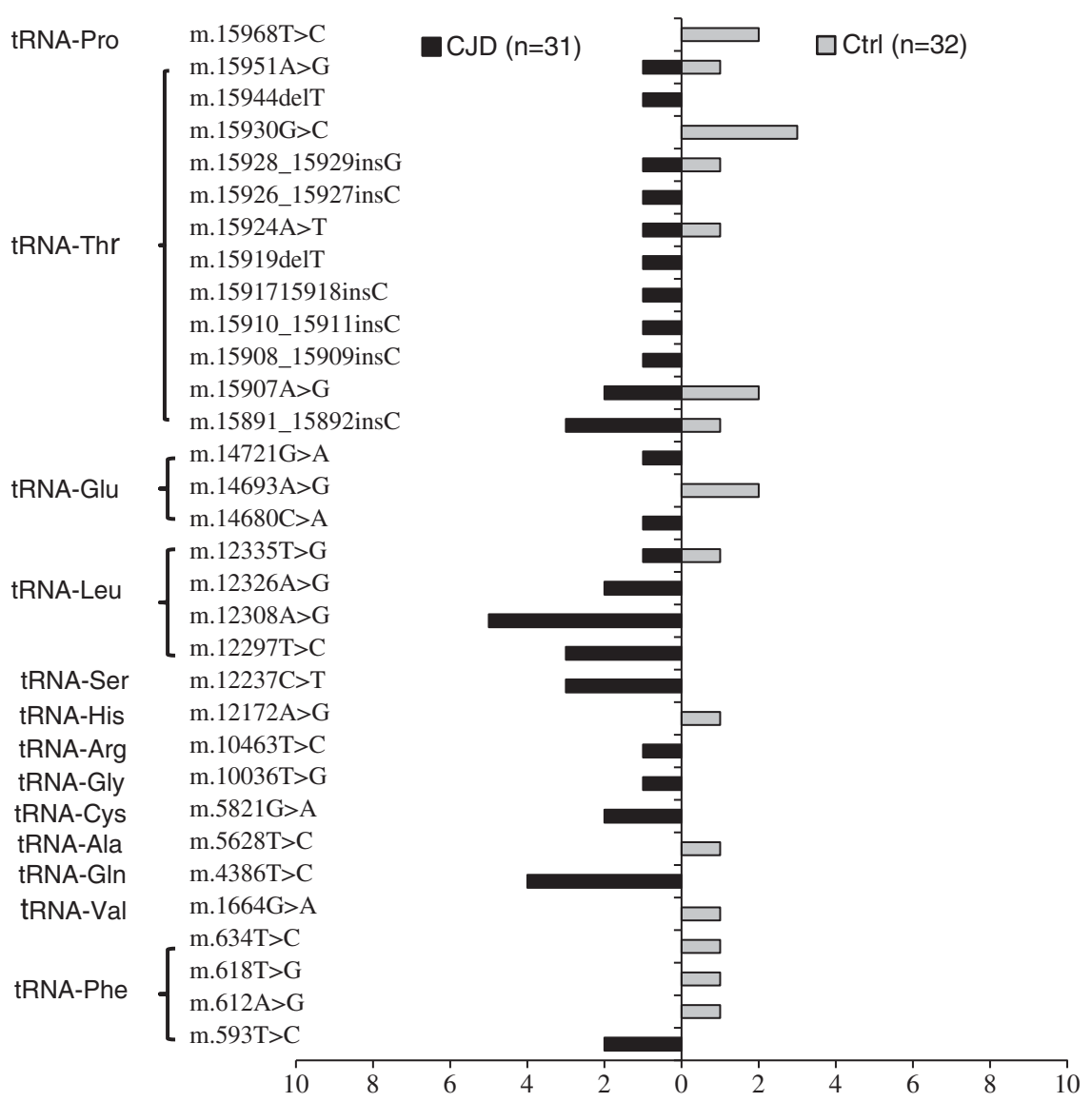

Figure 2 The numbers of the mutations in mtDNA tRNA between the groups of SCJD and controls.

\section{CONFLICT OF INTEREST}

The authors declare no conflict of interest.

\section{ACKNOWLEDGEMENTS}

This work was supported by Chinese National Natural Science Foundation Grant (81100980), China Mega-Project for Infectious Disease (2011ZX10004-101 and 2012ZX10004215), SKLID Development Grant (2012SKLID102, 2011SKLID302, 2011SKLID204, and 2011SKLID211).

1 Aguzzi A, Calella AM: Prions: protein aggregation and infectious diseases. Physiol Rev 2009; 89: 1105-1152.

2 Lukic A, Mead S: Genome wide association studies and prion disease. Prion 2011; 5: 154-160.

3 Mead S, Poulter M, Uphill J et al: Genetic risk factors for variant Creutzfeldt-Jakob disease: a genome-wide association study. Lancet Neurol 2009; 8: 57-66.

4 Owen F, Poulter M, Collinge J, Crow TJ: Codon 129 changes in the prion protein gene in Caucasians. Am J Hum Genet 1990; 46: 1215-1216.

5 Mead S, Whitfield J, Poulter $\mathrm{M}$ et al: A novel protective prion protein variant that colocalizes with kuru exposure. N Engl J Med 2009; 361: 2056-2065.

6 Shibuya S, Higuchi J, Shin RW, Tateishi J, Kitamoto T: Protective prion protein polymorphisms against sporadic Creutzfeldt-Jakob disease. Lancet 1998; 351: 419.

7 Mead S, Mahal SP, Beck J et al: Sporadic-but not variant-Creutzfeldt-Jakob disease is associated with polymorphisms upstream of PRNP exon 1. Am J Hum Genet 2001; 69: 1225-1235.

8 Sakaguchi S: Molecular biology of prion protein and its first homologous protein. J Med Invest 2007; 54: 211-223.

9 Calero O, Bullido MJ, Clarimon J et al: Genetic cross-interaction between APOE and PRNP in sporadic Alzheimer's and Creutzfeldt-Jakob diseases. PLoS One 2011; 6: e22090.

10 Calero O, Bullido MJ, Clarimon J et al: A common BACE1 polymorphism is a risk factor for sporadic Creutzfeldt-Jakob disease. PLoS One 2012; 7: e43926.
11 Calero 0, Bullido MJ, Clarimon J et al: Genetic variability of the gene cluster CALHM 1-3 in sporadic Creutzfeldt-Jakob disease. Prion 2012; 6: 407-412.

12 Toescu EC, Myronova N, Verkhratsky A: Age-related structural and functional changes of brain mitochondria. Cell Calcium 2000; 28: 329-338.

13 Gray MW, Burger G, Lang BF: The origin and early evolution of mitochondria. Genome Biol 2001; 2: REVIEWS 1018.

14 Fliss MS, Usadel $\mathrm{H}$, Caballero $\mathrm{OL}$ et al: Facile detection of mitochondrial DNA mutations in tumors and bodily fluids. Science 2000; 287: 2017-2019.

15 Fariss MW, Chan CB, Patel M, Van Houten B, Orrenius S: Role of mitochondria in toxic oxidative stress. Mol Interv 2005; 5: 94-111.

16 Liu SS: Cooperation of a 'reactive oxygen cycle' with the $Q$ cycle and the proton cycle in the respiratory chain-superoxide generating and cycling mechanisms in mitochondria. J Bioenerg Biomembr 1999; 31: 367-376.

17 Navarro A, Boveris A: Brain mitochondrial dysfunction in aging, neurodegeneration, and Parkinson's disease. Front Aging Neurosci 2010; 2, pii 34.

18 Abu-Amero KK: Leber's hereditary optic neuropathy: the mitochondrial connection revisited. Middle East Afr J Ophthalmol 2011; 18: 17-23.

19 Emmanuele V, Sotiriou E, Rios PG et al: A novel mutation in the mitochondrial DNA cytochrome b gene (MTCYB) in a patient with mitochondrial encephalomyopathy, lactic acidosis, and strokelike episodes syndrome. J Child Neurol 2013; 28: 236-242.

20 Corral-Debrinski M, Horton T, Lott MT et al: Marked changes in mitochondrial DNA deletion levels in Alzheimer brains. Genomics 1994; 23: 471-476.

21 Egensperger R, Kosel S, Schnopp NM, Mehraein P, Graeber MB: Association of the mitochondrial tRNA(A4336G) mutation with Alzheimer's and Parkinson's diseases. Neuropathol Appl Neurobiol 1997; 23: 315-321.

22 Kumar P, Henikoff S, Ng PC: Predicting the effects of coding non-synonymous variants on protein function using the SIFT algorithm. Nat Protoc 2009; 4: 1073-1081.

23 Ng PC, Henikoff S: SIFT: Predicting amino acid changes that affect protein function. Nucleic Acids Res 2003; 31: 3812-3814.

24 Siskova Z, Mahad DJ, Pudney C et al: Morphological and functional abnormalities in mitochondria associated with synaptic degeneration in prion disease. Am J Patho 2010; 177: 1411-1421.

25 Cali T, Ottolini D, Brini M: Mitochondrial $\mathrm{Ca}(2+)$ and neurodegeneration. Cell Calcium 2012; 52: 73-85. 
26 Singh N, Singh A, Das D, Mohan ML: Redox control of prion and disease pathogenesis. Antioxid Redox Signal 2010; 12: 1271-1294.

27 Mattei V, Matarrese P, Garofalo T et al: Recruitment of cellular prion protein to mitochondrial raft-like microdomains contributes to apoptosis execution. Mol Biol Cell 2011; 22: 4842-4853.

28 Coskun PE, Beal MF, Wallace DC: Alzheimer's brains harbor somatic mtDNA control-region mutations that suppress mitochondrial transcription and replication. Proc Natl Acad Sci USA 2004; 101: 10726-10731.

29 Ruiz-Pesini E, Mishmar D, Brandon M, Procaccio V, Wallace DC: Effects of purifying and adaptive selection on regional variation in human mtDNA. Science 2004; 303: 223-226.

30 Maruszak A, Canter JA, Styczynska M, Zekanowski C, Barcikowska M: Mitochondrial haplogroup $\mathrm{H}$ and Alzheimer's disease-is there a connection? Neurobiol Aging 2009; 30: $1749-1755$.

31 van der Walt JM, Dementieva YA, Martin ER et al: Analysis of European mitochondrial haplogroups with Alzheimer disease risk. Neurosci Lett 2004; 365: 28-32.

32 Martinez-Redondo D, Marcuello A, Casajus JA et al: Human mitochondrial haplogroup $\mathrm{H}$ : the highest VO2max consumer-is it a paradox? Mitochondrion 2010; 10: 102-107.
33 Baracca A, Solaini G, Sgarbi G et al: Severe impairment of complex I-driven adenosine triphosphate synthesis in Leber hereditary optic neuropathy cybrids. Arch Neurol 2005; 62: 730-736.

34 Morris AA, Leonard JV, Brown GK et al: Deficiency of respiratory chain complex I is a common cause of Leigh disease. Ann Neurol 1996; 40: 25-30.

$35 \mathrm{Lim} \mathrm{BC}$, Park JD, Hwang $\mathrm{H}$ et al: Mutations in ND subunits of complex I are an important genetic cause of childhood mitochondrial encephalopathies. J Child Neurol 2009; 24: 828-832.

36 Shadel GS, Clayton DA: Mitochondrial DNA maintenance in vertebrates. Annu Rev Biochem 1997; 66: 409-435.

37 Majamaa K, Rusanen H, Remes AM, Pyhtinen J, Hassinen IE: Increase of blood $\mathrm{NAD}+$ and attenuation of lactacidemia during nicotinamide treatment of a patient with the MELAS syndrome. Life Sci 1996; 58: 691-699.

38 Rego-Perez I, Fernandez-Moreno M, Fernandez-Lopez C, Arenas J, Blanco FJ: Mitochondrial DNA haplogroups: role in the prevalence and severity of knee osteoarthritis. Arthritis Rheum 2008; 58: 2387-2396.

39 Rollins B, Martin MV, Sequeira PA et al: Mitochondrial variants in schizophrenia, bipolar disorder, and major depressive disorder. PLoS One 2009; 4: e4913.

Supplementary Information accompanies this paper on European Journal of Human Genetics website (http://www.nature.com/ejhg) 